\title{
Management of Musculoskeletal Involvement in Systemic Sclerosis
}

\section{Philip Clements, MD, MPH}

\author{
Address \\ Professor Emeritus, David Geffen School of Medicine at UCLA, 200 Medical Plaza, \\ Rm 365-C, Los Angeles, CA, 90095, USA \\ Email: pclements@mednet.ucla.edu
}

Published online: 2 February 2016

(C) Springer International Publishing AG 2016

This article is part of the Topical Collection on Scleroderma

Keywords Systemic sclerosis $\cdot$ Scleroderma $\cdot$ Arthritis $\cdot$ Tendon friction rubs $\cdot$ Synovitis $\cdot$ Tender joint counts $\cdot$ Swollen joint counts · Bursitis - Fibromyalgia - Triple therapy for RA $\cdot$ Rituximab $\cdot$ Methotrexate

\section{Opinion statement}

Musculoskeletal (MSK) pain is a frequent complaint (between $40-80 \%$ of SSc patients) of patients with systemic sclerosis (SSC) and is most problematic in patients with early diffuse SSc. There are several MSK pain syndromes that can be seen in SSc: seronegative polyarthritis, rheumatoid arthritis, tendonitis, fibromyalgia and other MSK pain syndromes. There are, however, few systemically performed studies that show us the best ways to evaluate or to treat these syndromes. This article presents an expert's opinion about how to evaluate these pain syndromes and how best to treat them, given the limited amount of scientific data that are available.

\section{Introduction}

Musculoskeletal (MSK) pain is a frequent complaint of patients with systemic sclerosis (SSc) (between $40-80 \%$ of SSc patients) and is most problematic in patients with early diffuse SSc $[1 \bullet]$. Unfortunately there are virtually no systematic studies of the causes or the management of MSK involvements in SSc and with few exceptions there have been no controlled trials to determine what are and should be the best strategies for managing MSK pain and synovitis in patients with SSc.
A number of cross-sectional studies have been reported over the last few decades which give us a rough idea of how extensive the problem is. Richards reported patients' perceptions: stiff joints were noted in $79 \%$ of SSc patients, joint pain in $75 \%$, and generalized fatigue in $75 \%$ [2]. In a second study, hand dexterity was reduced to 68$80 \%$ and grip force to $46-65 \%$ compared to normals [3], while in a third study impairment of hand function (as assessed by the Cochin Hand 
Function Scale) was higher than in RA or OA [4].

Although the pain may not localize well enough to attribute it to a particular anatomic location, there are several MSK pain syndromes that can be seen in SSc: 1. Tendonitis $[5,6]$
2. Rheumatoid arthritis [7]
3. Polyarthritis (not RA) [8]
4. Fibromyalgia [9]
5. Other MSK syndromes

\section{Descriptions of the types of MSK pain syndromes seen in SSC}

Tendonitis: Although "tendonitis" in the generic sense occurs in many locations in SSc, the more unique form is characterized by the term "tendon friction rubs." These rubs may go unnoticed by the patient but more frequently the patients are aware of pain in that area and may even acknowledge a sense of "scraping" or "rubbing" when the joint moves $[5,6]$. The friction rub's underlying pathology is related to inflammatory fibrinous deposits on the surface of tendon sheaths and around the tendon. The areas that are most typically involved are the triceps, extensor, and flexor tendons of the wrist, patellar tendons, and the posterior and anterior tendons of the ankles. Other areas in which rubs can be found include subscapular, lateral trochanter, and paraspinal areas.

Rheumatoid arthritis: These are SSc persons with inflammatory polyarthritis who have RF and/or anti-cyclic citrullinated peptide antibodies ( $\mathrm{x}-\mathrm{CCP} \mathrm{ab}$ ) in addition to the clinical appearance of RA. Some of these patients develop erosive, destructive joint disease and a few develop a resorptive polyarthropathy resembling arthritis mutilans. The exact prevalence of these patients is estimated to be between $5-10 \%$ in the first 5 years of SSc disease.

Polyarthritis (non-RA): These are SSc persons with a polyarthralagia/polyarthritis who do not have RF or anti-CCP antibodies but still have polyarthritic complaints. Some may develop erosive destructive joint changes (particularly in the hands) while smaller numbers may develop resorptive arthropathy and arthritis mutilans $[8,10]$.

Fibromyalgia: Fibromyalgia is a syndrome-not a disease. It is characterized as a myofascial pain syndrome that involves many areas of the body ( 3 of 4 quadrants: above and below the waist and right and left of the spine) along with $\geq 11$ of 18 FMS points [9]. Although there are few published data on the prevalence of FMS in SS, a post hoc analysis of a previously published data set [11], Malcarne (unpublished observations) revealed that $18.6 \%$ of the 102 SSc patients examined met the 1990 classification criteria for FMS (predominantly patients with early diffuse SSC).

Other MSK syndromes: These other miscellaneous pain syndromes include carpal tunnel syndrome, de Quervain's tendonitis, trochanteric and gluteus medius bursitis (lateral hip), anserine bursitis (medial knee), olecranon bursitis, epicondylitis (lateral more than medial), and rotator cuff tendonitis to name a few. 


\section{Measurement of outcomes that can be used in the clinic to assess disease activity}

\section{Clinical measures}

The available literature regarding outcomes and evaluations of therapy in the musculoskeletal involvement by SSc is non-uniform [1•]. One might think that the seven core arthritis measures [12] used in assessing the response in Rheumatoid Arthritis could be borrowed directly for use in the examination of SSc patients with polyarthritis. However of the seven, only the function assessments have undergone validation in SSc, using the OMERACT filter criteria. These validated function assessments include the Disability Index of the Health Assessment Questionnaire (HAQ-DI), the Cochin Hand Function Scale and the Hand Mobility in Scleroderma (HAMIS) assessment, all of which address function:

1. The 20-item patient self-assessed HAQ-DI is the most widely employed and reported measure for assessing overall function in SSc. In addition nearly half of the 20 questions relate to hand function $[13,14]$.

2. Cochin Hand Function Scale: This is a patient selfreport questionnaire that assesses hand function in SSc [4, 14] (Table 1 displays the full questionnaire).

3. HAMIS: This is a performance-based (trained physical and/or occupational therapist is usually required although clinical metricians or physicians can be trained to perform the measure) evaluation of patient's function $[15,16]$. The instrument assesses function in the hand in SSc patients.

Validation aside, the other six RA core variables might have some utility in assessing the remaining six RA core measures, although none of them have been validated for use in SSc: tender joint count and swollen joint counts (28 joints particularly), indices of inflammation (ESR, CRP), the 10-cm Visual Analogue Scale (VAS) for pain, and the $10-\mathrm{cm}$ visual analogue scale (VAS) for patient global arthritis activity and the 10-cm VAS for physician global arthritis activity scales.

\section{Radiographs}

Plain X-ray films may be of some utility for evaluating the propensity to bone destruction of the patient's arthritic complaints: for example, erosive bone disease, resorptive arthropathy, and arthritis mutilans. The technique is not, however, good for picking up soft-tissue disease.

Ultrasound (MSUS) and magnetic resonance imaging (MRI)

The literature supporting the use of these imaging techniques in SSc is in its infancy; but already it is clear that either or both imaging techniques are much better at picking up synovitis, tendonitis, tenosynovitis and other soft-tissue disease than the clinical exam in SSc [1•]. In one study [17], for example, the baseline MSUS identified tenosynovitis in $8(47 \%)$ and synovitis in $1(6 \%)$ of the 17 SSc patients who were examined for complaints of arthralgia and in $6(46 \%)$ and $3(23 \%)$ of the 12 SSc patients who returned for a second MSUS. In the same study [17], all eight patients who were examined by MRI showed evidence of synovial inflammation in the form of synovitis in eight (100\%) of the eight and tenosynovitis in seven ( $88 \%)$ of the eight patients. Bone edema was seen in five ( $63 \%)$ of the eight and erosions in six $(75 \%)$ of the eight patients.

Even with the few studies published of MSUS and MRI in SSc-arthritis, performed either at baseline or over time, these imaging studies will likely provide much more detail of the MSK involvement by SSc that can employed to improve the clinical management of these patients.

\section{Summary of the most reasonable outcome measures that can be used in the clinical management of SSC patients with polyarthritis}

1. At baseline, examine the patient. Consider X-rays, ultrasound/MRI assessments of the fingers, hands and wrist joints and especially their surrounding soft tissues. Draw rheumatoid factor, anti-CCP antibodies, ESR, and CRP.

2. At periodic times assess:

(a) Joint tenderness counts (28 joints, as in RA).

(b) Joint swelling counts (28 joints, as in RA); also realize that thickened skin on the hands and fingers may make this assessment difficult.

(c) 10-cm visual activity scales (VAS) to assess patient's pain, the patient's 10-cm VAS for global assessment of how their arthritis is doing, and the doctor's 10-cm global assessment of how the patient's arthritis is doing. 


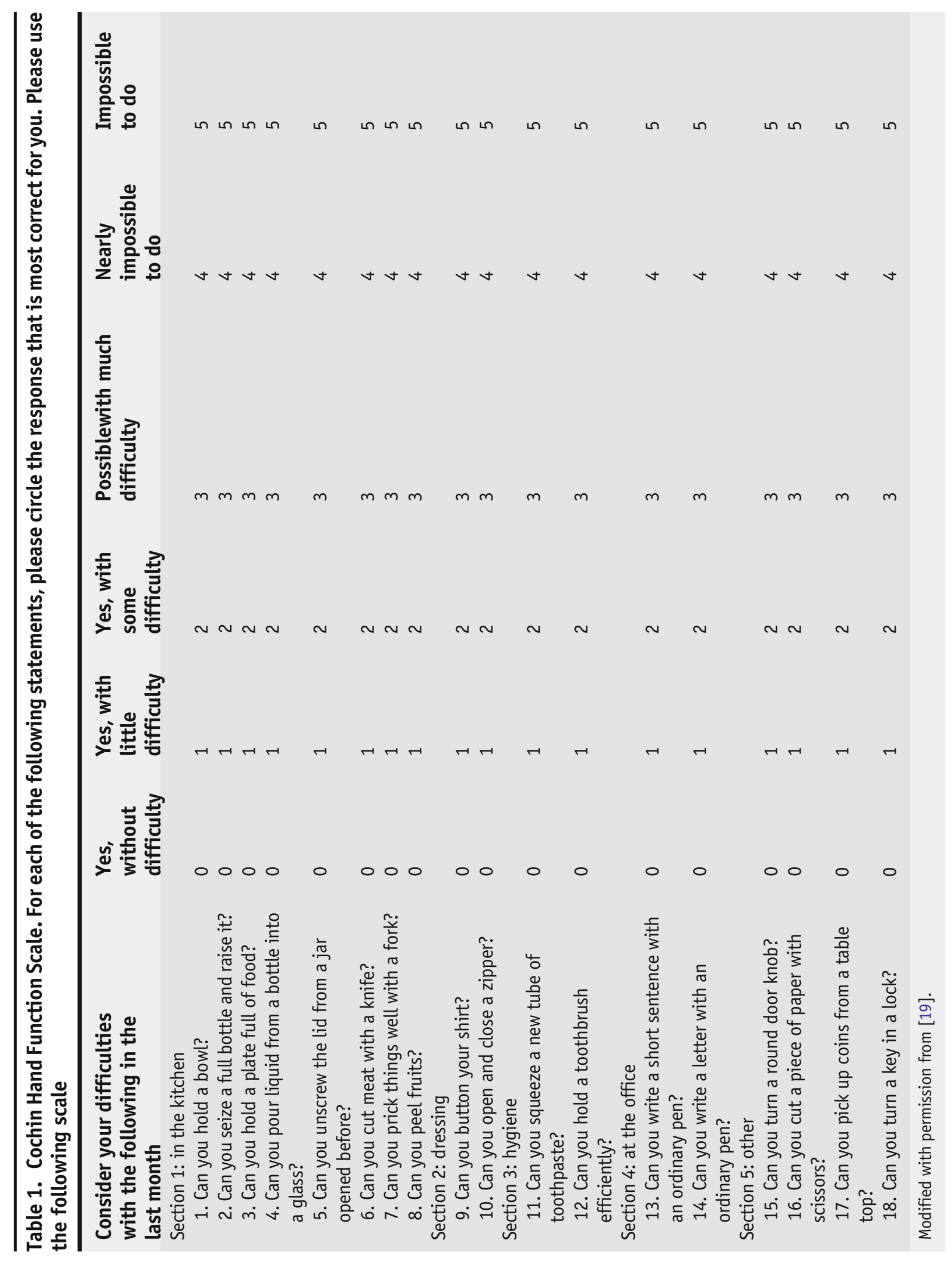


(d) The Cochin Hand Function Scale (Table 1) is particularly useful here as a measure of function because it is focused on hand function and how that function may change over time. It is patientderived; its questions are readily understood and can be completed in less than $5 \mathrm{~min}$. It may, however, be confounded by the fibrotic associated finger contractures that are part of SSc.

(e) It is less clear how the ESR and CRP may change in meaningful ways in SSc-arthritis but it may be worth following in given individuals.

\section{Management of individual MSK syndromes}

Seronegative Polyarthritis Walker et al [18 $\bullet$ canvassed 117 SSc experts in a 3-part internet exercise about how they would treat the entity of "inflammatory polyarthritis" in SSc. There was no written definition of inflammatory polyarthritis provided to the participants, and as such the participants were allowed to use their own criteria for making that diagnosis. 59 experts (43\% of the initial 117) completed all three parts of the survey. In the survey the first line treatments reported by participants included methotrexate in $60 \%$, corticosteroids in $30 \%$, and hydroxychloroquine in $28 \%$. If the patient was not improved enough with the initial treatments, $75 \%$ of the participants reported that they would add a second agent while $25 \%$ reported they would switch to a second, different agent. The second line treatments chosen by the participants included methotrexate in $54 \%$, corticosteroids in $37 \%$, hydroxychloroquine in $31 \%$, and biologics (including TNF-inhibitors [TNFi]) in $20 \%$.

A second approach to treatment (Fig. 1) has been suggested that divides SSc patients with inflammatory polyarthritis by phenotype into those who look more like:

1. Erosive polyarthritis that is seronegative (rheumatoid factor [RF] and cyclic citrullinated peptide antibody [anti-CCP] tests negative) that can look like destructive RA, psoriatic arthritis and/or arthritis mutilans. In many respects they can be treated like rheumatoids even though efficacy in SSc has not been established.
2. Non-erosive polyarthritis (rheumatoid factor [RF] and cyclic citrullinated peptide antibody [antiCCP] tests negative) that can be treated much as a lupus-like arthritis.

In this scheme, all polyarthritis could/should be treated with non-steroidal anti-inflammatory agents (as tolerated by the kidneys and gastrointestinal tract), low-dose corticosteroids (with the exception of early diffuse SSc [ $\leq 5$ years from SSc onset] who may be at increased risk of renal crisis if managed on corticosteroids at any dose), local corticosteroid injections, and hand therapy to stretch contractured joints, improve range of motion, and potentially minimize future contractures and functional disability.

As far as treatment of the polyarthritic phenotypes is concerned, we should keep in mind that the following treatment strategies have not been scientifically tested in SSc per se even though scientifically they have been found effective in RA. At this point we are forced to accept expert opinion for the following recommendations: The erosive patients (even if they are RF and/or anti-CCP negative) might be started on methotrexate (MTX). If MTX is not effective enough, leflunomide, triple therapy or a tumor necrosis factor inhibitor (TNFi) might be added. If these strategies fail then moving on to other biologics might be reasonable. The drugs listed in the figure have by and large been shown in RA to be bone preservers, and that can be the rationale for using them in this situation. Again we should keep in mind that there is little science in the use of these agents in SSc.

The same caveat holds true about the lack of scientific basis for the management of the nonerosive lupus-like polyarthritis in SSc as well. Potential treatments are listed in Fig. 1. By and large the drugs listed in the non-erosive pathway have failed to show convincing evidence that they are significant bone preservers in RA. They can be used separately or in combinations as they often are in practice.

Seropositive Polyarthritis or RA (RF and Anti-CCP Antibody Positive) The presence of seropositive RA (+ anti-CCP antibody) in association with SSc suggests a true overlap syndrome. Its management should 


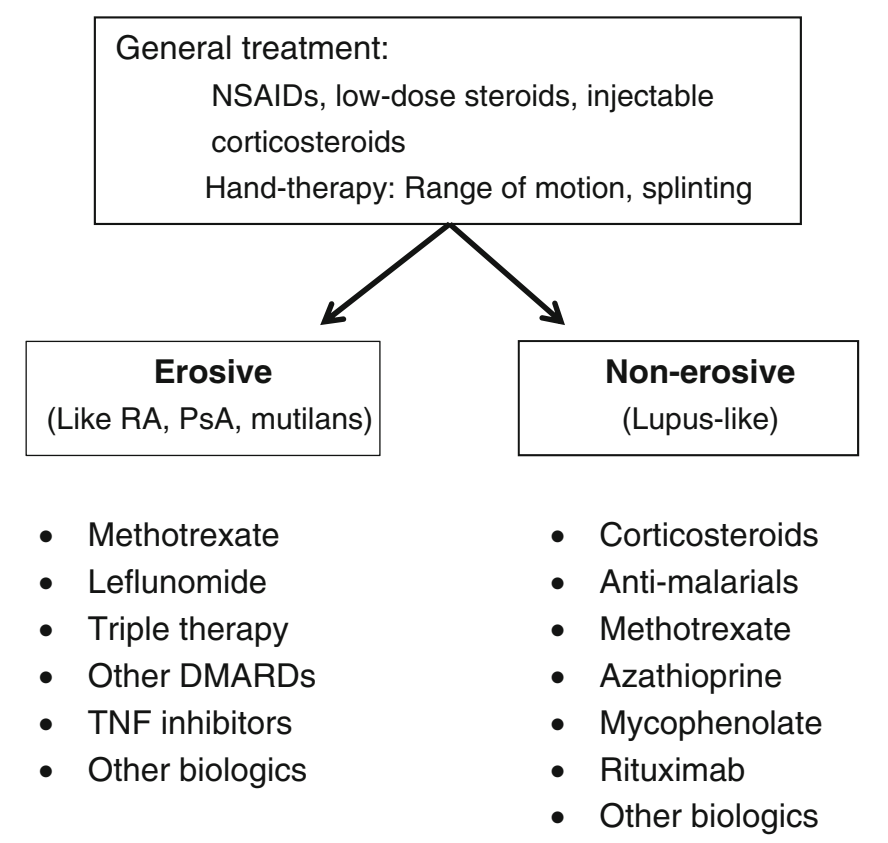

Fig. 1. Suggested algorithm for treatment of SSc-arthritis by erosive versus non-erosive polyarthritis.

be driven by the scientific precepts that have developed for the management of RA. I will not discuss this entity further.

Fibromyalgia As noted earlier in the introduction, about a fifth of SSc patients may have a fibromyalgia-like pain disorder at some point in their course. One useful treatment strategy is to decide who does and who does not respond to steroids:

1. Those who respond well to small doses of prednisone (5-7.5 mg daily or less) or other shortacting corticosteroid in equivalent doses. Caution: avoid the use of prednisone in patients with diffuse SSc of less than 5 years of SSc.

2. Those who do not respond to small doses of prednisone.

My recommendations here are to introduce to the patient the concept that about $20 \%$ of SSC patients have this myofascial pain syndrome called FMS and that we need to address the pain and fatigue concerns that plague these patients, almost as a separate overlapping disorder. Essentially I treat FMS as a separate entity within the larger context of SSc. The patient should be reassured that when the pain and fatigue flair, it is usually not that the SSc is getting worse-only that their FMS has flared.

My management principles for SSc-associated FMS are those of many experienced practitioners who see and treat FMS. I do, however, often employ a short trial (2-4 weeks) of lowdose prednisone to determine if the FMS-like disorder is really an inflammatory process that just looks like FMS. If the patient responds, then I will try to extend the time for using steroids and try to taper the prednisone to the lowest effective dose. Whether the patient does or does not respond to steroids, I otherwise treat them as if they had FMS on a noninflammatory basis.

Miscellaneous Soft-Tissue Involvements Including Tendonitis, Tenosynovitis, and Bursitides MSUS/MRI appear to be useful tools for detecting the presence of inflammatory and structural abnormalities involving both joints and soft tissues [1•]. The swollen and inflamed soft tissues that are part of SSc are often more obvious and better defined by MSUS/MRI than are apparent by clinical examination. If the health provider has any question 
about how involved seemingly simple soft tissues are, then MSUS and MRI may be helpful in demonstrating the involvements that can be managed appropriately. Many of the soft tissue involvements that are uncovered by MSUS/MRI are as treatable as they might be in the general rheumatic disease population: NSAIDS, softtissue steroid injections, physical and occupational therapy, splinting, etc.

\section{Summary}

Although the science of assessing and treating MSK involvements in SSc is in its infancy, there are strategies that many experts feel can help the symptomatic SSc patient with arthritic complaints. The present treatments, largely based on expert opinion, have been presented in the discussion above. The diagnostic criteria and the outcomes to therapy need to be evaluated and validated in prospective controlled trials.

\section{Compliance with Ethical Standards}

\section{Funding}

This work was supported by grants from the NHLBI/NIH: R01 HL089758 and R01 HL089901.

\section{Conflict of Interest}

Philip Clements declares that he has no conflicts of interest.

\section{Human and Animal Rights and Informed Consent}

This article does not contain any studies with human or animal subjects performed by the author.

\section{References}

Papers of particular interest, published recently, have been highlighted as:

- $\quad$ Of importance

1. $\quad$ Clements PJ, Allanore Y, Khanna D, Singh M, Furst DE. Arthritis in systemic sclerosis: systematic review of the literature and suggestions for the performance of future clinical trials in systemic sclerosis arthritis. Semin Arthritis Rheum. 2012;41:801-14.

This systematic literature review revealed that there was very little in the way of systematically performed studies of outcomes or of therapies that could impact polyarthritis or other musculoskeletal manifestation of systemic sclerosis.

2. Richards HL, Herrick AL, Griffin K, Gwilliam PDH, Loukes J, Fortune DG. Systemic sclerosis: patients' perceptions of their condition. Arthritis Care Res. 2003;49:689-96.

3. Sandqvist G, Eklund M, Akesson A, Nordenskiöld U. Daily activities and hand function in women with scleroderma. Scand J Rheumatol. 2004;33:102-7.
4. Brower LM, Poole JL. Reliability and validity of the Duruoz Hand Index in persons with systemic sclerosis (scleroderma). Arthritis Rheum. 2004;51:805-9.

5. Steen VD, Medsger Jr TA. The palpable tendon friction rub: an important physical examination finding in patients with systemic sclerosis. Arthritis Rheum. 1997;40:1146-51.

6. Khanna PP, Furst DE, Clements PJ, Maranian P, Indulkar L, Khanna D. D-Penicillamine investigators. Tendon friction rubs in early diffuse systemic sclerosis: prevalence, characteristics and longitudinal changes in a randomized controlled trial. Rheumatology (Oxford). 2010;49:955-9.

7. Morita Y, Muro Y, Sugiura K, Tomita Y. Anti-cyclic citrullinated peptide antibody in systemic sclerosis. Clin Exp Rheumatol. 2008;26:542-7. 
8. Avouac J, Guerini H, Wipff J, Assous N, Chevrot A, Kahan A, et al. Radiological hand involvement in systemic sclerosis. Ann Rheum Dis. 2006;65:1088-92.

9. Wolfe F, Smythe HA, Yunus MB, Bennett RM, Bombardier C, Goldenberg DL, et al. The American College of Rheumatology 1990 criteria for the classification of fibromyalgia: report of the multicenter criteria committee. Arthritis Rheum. 1990;33:160-72.

10. Bassett LW, Blocka KL, Furst DE, Clements PJ, Gold RH. Skeletal findings in progressive systemic sclerosis (scleroderma). AJR Am J Roentgenol. 1981;136:1121-6.

11. Malcarne VL, Hansdottir I, McKinney A, Upchurch R, Greenbergs HL, Henstorf GH, et al. Medical signs and symptoms associated with disability, pain, and psychosocial adjustment in systemic sclerosis. J

Rheumatol. 2007;34:359-67.

12. Felson DT, Anderson JJ, Boers M, Bombardier C, Chernoff M, Fried B, et al. The American College of Rheumatology preliminary core set of disease activity measures for rheumatoid arthritis clinical trials. The Committee on Outcome Measures in Rheumatoid Arthritis Clinical Trials. Arthritis Rheum. 1993;36:729-40.

13. Steen VD, Medsger Jr TA. The value of the health assessment questionnaire and special patient-generated scales to demonstrate change in systemic sclerosis patients over time. Arthritis Rheum. 1997;40:1984-91.

14. Clements PJ, Wong WK, Hurwitz EL, Furst DE, Mayes $\mathrm{M}$, White $\mathrm{B}$, et al. Correlates of the disability index of the health assessment questionnaire: a measure of functional impairment in systemic sclerosis. Arthritis Rheum. 2001;42:2372-80.

15. Sandqvist G, Eklund M. Validity of HAMIS: a test of hand mobility in scleroderma. Arthritis Care Res. 2000;13:382-7.

16. Sandqvist G, Eklund M. Hand mobility in scleroderma (HAMIS) test: the reliability of a novel hand function test. Arthritis Care Res. 2000;13:369-74.

17. Chitale S, Ciapetti A, Hodgson R, Grainger A, O'Connor P, Goodson NJ, et al. Magnetic resonance imaging and musculoskeletal ultrasonography detect and characterize covert inflammatory arthropathy in systemic sclerosis patients with arthralgia. Rheumatology (Oxford). 2010;49:2357-61.

18. Walker KM, Pope J. Participating members of the scleroderma clinical trials consortium (SCTC), Canadian scleroderma research group. Treatment of systemic sclerosis complications: what to use when first-line treatment fails-a consensus of systemic sclerosis experts. Semin Arthritis Rheum. 2012;42:42-55.

This was not a scientific study of the practices of scleroderma experts from around the world. Rather, it was questioning of physicians of what the physicians themselves thought about how they treated polyarthritis and other musculoskeletal manifestations as they occur in systemic sclerosis.

19. Duruöz MT, Poiraudeau S, Fermanian J, Menkes CJ, Amor B, Dougados M, et al. Development and validation of a rheumatoid hand functional disability scale that assesses functional handicap. J Rheumatol. 1996;23:1167-72. 